\title{
Anaesthetic, analgesic and cardiorespiratory effects of intramuscular medetomidine-ketamine combination alone or with morphine or tramadol for orchiectomy in cats
}

\author{
Gareth E Zeiler*, Brighton T Dzikiti*, Geoffrey T Fosgate†, Frik G Stegmann*, Frans J Venter* \\ \& Eva Rioja* \\ *Department of Companion Animal Clinical Studies, Faculty of Veterinary Science, University of Pretoria, \\ Onderstepoort, South Africa \\ $\dagger$ Department of Production Animal Studies, Faculty of Veterinary Science, University of Pretoria, Onderstepoort, \\ South Africa
}

Correspondence: Gareth E Zeiler, Department of Companion Animal Clinical Studies, Faculty of Veterinary Science, University of Pretoria, P.Bag X04, Onderstepoort, Gauteng, 0110, South Africa. E-mail: gareth.zeiler@up.ac.za

Runner: Three medetomidine-ketamine combinations in cats

\begin{abstract}
Objectives To compare the anaesthetic, analgesic and cardiorespiratory effects of intramuscular (IM) medetomidine and ketamine administered alone or combined with morphine or tramadol, for orchiectomy in cats.

Study Design Randomised, blinded, prospective clinical study. Animals Thirty client-owned cats.
\end{abstract}


Materials and Methods Cats ( $\mathrm{n}=10$ in each group) received a combination of medetomidine $\left(60 \mu \mathrm{g} \mathrm{kg}^{-1}\right)$ and ketamine $\left(10 \mathrm{mg} \mathrm{kg}^{-1}\right)$ alone (MedK); combined with morphine $\left(0.2 \mathrm{mg} \mathrm{kg}^{-1}\right)$ (MedKM), or combined with tramadol (2 $\left.\mathrm{mg} \mathrm{kg}^{-1}\right)$ (MedKT) IM. Time of induction, surgical and recovery events were recorded, and physiological parameters measured and recorded. Analgesia was evaluated with a visual analogue scale, a composite scoring system and the von Frey mechanical threshold device every hour from three to eight hours post-drug administration. injection. Data were analyzed with a linear mixed model, Kruskal Wallis or Chi-square tests $(\mathrm{p}<0.05)$.

Results Median (range) induction and recovery times (minutes) were not significantly $(\mathrm{P}=0.125)$ different among the three combinations: 5.6 (2.7- 8.0), 7.4 (5.1-9.6) and 8.0 (5.8-14.9) for induction and 128.5 (95.1-142.8), 166.4 (123.1-210.0) and 142.9 (123.4-180.2) for recovery, with MedK, MedKT and MedKM, respectively. Two cats in group MedKM required alfaxalone for intubation. In all groups, 3 or 4 cats required additional isoflurane for surgery, arterial oxygen tension (mean \pm SD: $66 \pm 2 \mathrm{mmHg}$ ) was low, and, surgery increased systolic arterial blood pressure $(\mathrm{p}<0.001)$, haemoglobin saturation $(\mathrm{p}<0.001)$, respiratory $(\mathrm{p}=0.003)$ and heart rates $(\mathrm{p}=0.002)$, and decreased end-tidal carbon dioxide $(\mathrm{p}=0.003)$. Pain scores did not differ significantly between groups. Von Frey responses decreased over time, changes over time varied by treatment $(\mathrm{p}<0.001)$, the MedK group returning to baseline values more rapidly than MedKM and MedKT. None of the cats required rescue analgesics.

Conclusion and Clinical Relevance All three protocols provide adequate anaesthesia and analgesia for orchiectomy in cats. However, rescue intervention to maintain surgical anaesthesia such as isoflurane may be required in some cats. Oxygen supplementation is advised. Keywords feline, medetomidine, ketamine, morphine, tramadol, anaesthesia, orchiectomy 


\section{Introduction}

Many different combinations of intramuscularly injected drugs are used in private veterinary practices and welfare clinics to anaesthetise healthy domestic cats for routine surgical procedures, but the majority are based on ketamine in combination with an alpha 2 adrenoceptor agonist such as medetomidine. The Confidential Enquiry into Perioperative Small Animal Fatalities (CEPSAF) study showed that the overall risk of anaesthetic and sedation-related death in healthy cats was greater than that of dogs (Brodbelt et al. 2007; Brodbelt et al. 2008). The high incidence of anaesthetic accidents reported strengthens the need to investigate whether there are anaesthetic combinations that are more reliable and predictable for routine procedures in cats. Cats are unique and it is difficult to accurately extrapolate research data on drug pharmacodynamics, pharmacokinetics, efficacy and pain scoring systems from other species (Taylor et al. 2004).

Routine use of analgesics in cats is often neglected due to fears of undesirable outcomes. However, morphine (Wiese et al. 2007) and tramadol (Brondani et al. 2009; Cagnardi et al. 2011; Castro et al. 2009) have been investigated in cats and the two agents have been shown to provide comparable analgesia in a number of research and clinical trials. There is no report, however, of their use in cats within a medetomidine and ketamine combination under clinical conditions, either in relation to the cardiopulmonary effects of their combinations or as to the provision of peri-operative analgesia. The peri-operative analgesic effect of a drug is difficult to assess or quantify, particularly in the domestic cats as there are few published reports validating pain scoring systems in this species (Brondani et al. 2011).

The present study aimed to compare the anaesthetic, analgesic and cardiorespiratory effects of a combination of medetomidine and ketamine alone or in combination with morphine 
or tramadol administered by the intramuscular route to induce surgical anaesthesia for routine orchiectomy in domestic cats.

\section{Materials and Methods}

The study was approved by the Animal Ethics Committee of the University of Pretoria (Protocol V044-11). All owners gave their consent. The study was conducted at the University of Pretoria; this is situated at a height of 1252 metres; thus normal barometric (atmospheric) pressure ranges from 651 to $668 \mathrm{mmHg}(86.8$ to $89.1 \mathrm{kPa})$.

The sample size for the study was calculated based to detect an increase in heart rate of $10 \%$ over baseline during the surgical procedure, assuming a standard deviation of $10 \%$, a power of $80 \%$, and an alpha error of $5 \%$. Thus 30 healthy intact male cats were enrolled, ten cats in each of the three treatment groups. On admission (day 1), all cats underwent a comprehensive physical examination, haematology (complete blood count), serum biochemistry (total serum protein, creatinine) and echocardiography to ensure they satisfied the profile of American Society of Anesthesiologists physical status I.

\section{Anaesthetic protocol}

The cats were starved for eight hours and water was withheld for two hours before the procedure. They then were assigned randomly (Randomisation Allocation Software Version 1, University of Medical Science, Iran) to one of following three treatment groups.

- MedK group: medetomidine $\left(60 \mu \mathrm{g} \mathrm{kg}^{-1}\right.$; Domitor $1 \mathrm{mg} \mathrm{mL}^{-1}$; Pfizer Laboratories; South Africa) and ketamine (10 $\mathrm{mg} \mathrm{kg}^{-1}$; Ketamine $100 \mathrm{mg} \mathrm{mL}^{-1}$; Intramed; South Africa). 
- MedKM group: medetomidine $\left(60 \mu \mathrm{g} \mathrm{kg}^{-1}\right)$, ketamine $\left(10 \mathrm{mg} \mathrm{kg}^{-1}\right)$ and morphine $(0.2$ $\mathrm{mg} \mathrm{kg}^{-1}$; Morphine Sulphate $10 \mathrm{mg} \mathrm{mL}^{-1}$; Intramed; South Africa).

- MedKT group: medetomidine $\left(60 \mu \mathrm{g} \mathrm{kg}^{-1}\right)$, ketamine $\left(10 \mathrm{mg} \mathrm{kg}^{-1}\right)$ and tramadol $(2 \mathrm{mg}$ $\mathrm{kg}^{-1}$; TramaHexal $50 \mathrm{mg} \mathrm{mL}{ }^{-1}$; Hexal Pharma; South Africa).

All treatment drugs were mixed in a single syringe and administered as a single intramuscular (IM) injection into the lumbar epaxial group. The first author was unaware of the treatment used and captured all data during the study.

Induction phase

The time of IM injection of the treatment was recorded as time zero (T0). All time parameters were recorded using a stopwatch that was started immediately after injection. Anaesthetic induction time was calculated as time from $\mathrm{T} 0$ to loss of pedal reflex. Other recorded parameters during the induction phase included level of excitement, agitation or anxiety (pacing, excessive grooming of whole body) before and after the injection, nausea (lip licking and/or retching) and emesis. Once the pedal reflex was lost the cats were shaved in various regions to facilitate instrumentation, aseptic intravascular catheterisation and blood sampling. Thereafter the cats were moved to the operating theatre.

Maintenance phase

A cephalic venous cannula ( 22 gauge; $25 \mathrm{~mm}$ ) was placed. Following a single puff of local anaesthetic spray (Xylocaine $10 \mathrm{mg} / 0.1 \mathrm{~mL}$; AstraZeneca Pharmaceuticals (Pty) Ltd.; Wilmington DE, USA) over the arytenoid cartilages, orotracheal intubation with a size $4.0 \mathrm{~mm}$ uncuffed, polyvinylchloride endotracheal (ET) tube was performed. Glottis activity (presence or 
absence of partial or complete spasm) and ease of intubation (presence or absence of coughing or swallowing) were recorded. Cats in which the trachea could not be intubated due to a light anaesthetic plane received an intravenous (IV) dose of alfaxalone $\left(0.5-1 \mathrm{mg} \mathrm{kg}^{-1}\right.$; Alfaxan-CD RTU; Kyron Labratories; South Africa), titrated to effect to facilitate intubation. Systolic arterial blood pressure (SABP) was measured using a Doppler monitor (Ultrasonic Doppler Flow Detector Model 811-AL; Parks Medical Electronics; OR, USA) as previously described (Henik et al. 2005). The cuff (width of cuff was $40 \%$ diameter of limb) attached to a manual trigger manometer was placed on the mid-antebrachium. Doppler SABP readings were obtained three consecutive times and averaged for statistical analysis. Peripheral oxygen haemoglobin saturation $\left(\mathrm{SpO}_{2}\right)$ was measured by placing the pulse oximeter probe (Veterinary Pulse Oximeter/ $\mathrm{CO}_{2}$ Detector Model 9847V; Nonin Medical; MA, USA) on the tongue. Additionally monitored parameters included electrocardiogram (ECG), end-tidal carbon dioxide $\left(\mathrm{PE}^{\prime} \mathrm{CO}_{2}\right)$, oesophageal temperature (Temp), heart rate (HR) and respiratory rate $\left(f_{\mathrm{R}}\right)$; which were all obtained from a multi-parameter monitoring machine (Datex-Ohmeda S/5 Anesthesia Monitor; GE Healthcare; Finland). The multi-parameter monitoring machine made use of a side-stream gas analyser, sampling at $200 \mathrm{~mL}$ minute $^{-1}$ to determine partial pressures of respiratory gases. Physiological parameters were recorded at five minute intervals from instrumentation until 60 minutes after treatment injection (T60). All cats received an IV balanced isotonic crystalloid solution (10 mL kg ${ }^{-1}$ hour ${ }^{-1}$; Intramed Ringer-Lactate Solution; Intramed; South Africa) from the time the cephalic cannula was placed until T60. Normothermia was maintained by placing the cats on a pre-heated $\left(39.0^{\circ} \mathrm{C}\right)$ warm water blanket, wrapping them in a drape and using a forced air warming device. 
Simultaneous arterial and venous blood samples $(1 \mathrm{~mL}$ each $)$ were collected anaerobically into lithium heparin syringes via needle puncture of the femoral artery and from a jugular cannula (22gauge; $25 \mathrm{~mm}$ ), respectively. Samples were collected just prior to surgery while the cats were spontaneously breathing room air and analyzed ( $\alpha$-stat at $37.0^{\circ} \mathrm{C}$ ) within three minutes after collection (Rapidlab 348 System; Siemens; South Africa). The protocol stated that oxygen rescue was to be implemented after the arterial sample had been obtained if a cat had a consistently low $\mathrm{SpO}_{2}$ reading of less than $90 \%$ for longer than one minute or an arterial partial pressure of oxygen $\left(\mathrm{PaO}_{2}\right)$ less than $70 \mathrm{mmHg}(9.3 \mathrm{kPa})$. The alveolar partial pressure of oxygen $\left(\mathrm{P}_{\mathrm{A}} \mathrm{O}_{2}\right)$ was calculated in mmHg using the standard alveolar gas equation $\left[\mathrm{P}_{\mathrm{A}} \mathrm{O}_{2}=\mathrm{FiO}_{2}\left(\mathrm{P}_{\text {bar }}-\right.\right.$ $\left.\left.\mathrm{P}_{\mathrm{H} 2 \mathrm{O}}\right)-\mathrm{PaCO}_{2} / \mathrm{RQ}\right]$; using a mean barometric pressure $\left(\mathrm{P}_{\mathrm{bar}}\right)$ of $662 \mathrm{mmHg}$; a partial pressure of water $\left(\mathrm{P}_{\mathrm{H}_{2} \mathrm{O}}\right)$ of $47 \mathrm{mmHg}$ and a respiratory quotient (RQ) of 0.8 (Cinel et al. 1991). The arterial partial pressure of carbon dioxide $\left(\mathrm{PaCO}_{2}\right)$ and $\mathrm{PaO}_{2}$ of each cat were used accordingly in the alveolar gas equation and alveolar-arterial oxygen gradient $\left[\mathrm{P}(\mathrm{A}-\mathrm{a}) \mathrm{O}_{2}=\mathrm{PAO}_{2}-\mathrm{PaO}_{2}\right]$ calculations to compare differences among treatments.

Surgery phase

Orchiectomy was performed by the same experienced surgeon on all cats using a standardised technique. The time period from loss of pedal reflex to start of surgery was recorded. If the cat responded to the surgical stimulus by purposeful movement of the legs, neck or tail it was connected to a Mapleson D circuit and isoflurane (Isofor; Safeline Pharmaceuticals; South Africa) was administered in oxygen (1.0 L minute $\left.{ }^{-1}\right)$ via a precision vaporiser (Ohmeda Isotec 5; BOC Health Care; UK) at an initial vaporiser setting of $1 \%$, in order to deepen the plane of 
anaesthesia. Physiological parameters were recorded at critical surgical stages: plucking of scrotal hair, scrotal skin cuts, and tying-off of the spermatic cords and blood vessels.

Recovery phase

The cats were allowed to recover spontaneously after surgery without pharmacological antagonism of the injected drugs (medetomidine, morphine or tramadol). If cats were judged to be deeply anaesthetized or in danger of cardiovascular (atipamezole to reverse medetomidine) or respiratory distress (naloxone to reverse morphine or tramadol) then specific antagonists were administered and this action was recorded. Recovery time frames recorded included time to extubation, time to regain pedal reflex, and time to standing; all calculated from T0. Recovery time was calculated as the time from end of surgery to standing. Presence or absence of excitement, euphoria, nausea and emesis, sedation (heavy, moderate, mild) and pupil diameter (normal, mydriatic, miotic) were recorded hourly until 8 hours after T0.

\section{Pain Scoring}

A visual analogue scale (VAS; $100 \mathrm{~mm}$ line, $0=$ no pain, $100=$ worst possible imaginable pain) and a modified multidimensional composite pain scoring system (MCP; from $0=$ no pain to $23=$ maximal score) (Brondani et al. 2011) were used to assess postoperative pain at hourly intervals from 3 to 8 hours after T0. Buprenorphine $\left(0.02 \mathrm{mg} \mathrm{kg}^{-1}\right.$; Temgesic $0.3 \mathrm{mg} \mathrm{mL}^{-1} ; \mathrm{R} \& \mathrm{C}$ Pharmaceuticals; UK) was administered intramuscularly if a cat's score was $\geq 30 \%$ of the maximum possible score on either pain scoring system. Additionally, a rigid tip $(0.5 \mathrm{~mm}$ in diameter) mechanical threshold device (Electronic von Frey Anesthesiometer 2390 series; IITC Inc. Life Science; CA, USA) was used to assess level of response towards noxious mechanical 
stimulation unrelated to the surgical site. The pressure tip was applied to both sides of the midlateral thorax and analgesia assessment was repeated twice on each side. A noxious response was considered to be rapid movement of the head towards the stimulation site, vocalization, or aggression towards the device. The mechanical stimulus was stopped at the first indication of pain and the last reading (in grammes) was recorded. Two baseline mechanical threshold readings, one 24 hours and another one 2 hours before T0 were obtained and averaged for analysis. Post-operative mechanical threshold readings were obtained at 4,6 , and 8 hours after T0. All cats received a single dose of carprofen $\left(4 \mathrm{mg} \mathrm{kg}^{-1}\right.$; Rimadyl injectable; Pfizer Laboratories; South Africa) subcutaneously after the last data collection point, 8 hours after T0.

\section{Statistical Analysis}

Data were assessed for normality by the evaluation of descriptive statistics, plotting of histograms, and performing the Anderson-Darling test for normality. All data were presented as median and interquartile ranges (IQR) if one or more variable appeared to violate the normality assumption. Categorical data were compared among groups using chi-square tests followed by multiple pairwise comparisons using Fisher exact tests with Bonferroni correction of $\mathrm{p}$ values. Quantitative data were compared using Kruskal-Wallis tests followed by multiple pairwise Mann-Whitney U tests with Bonferroni correction. A linear mixed model approach was used to estimate the effect of treatment, time, and surgery on cardiorespiratory variables $\left(\mathrm{HR}, f_{\mathrm{R}}, \mathrm{SABP}\right.$, $\mathrm{PE}^{\prime} \mathrm{CO}_{2}, \mathrm{SpO}_{2}$ ), including a random effect for cat that assumed a first-order autoregressive correlation structure among observations. Mixed models were adjusted for the cat's body temperature and whether or not oxygen or isoflurane rescue was administered. Wilcoxon signed rank tests were used to compare multidimensional composite pain scores pre- and post-sedation. 
Data were analyzed using commercially available software (SPSS version 20.0; SPSS Inc;

Chicago, USA) and results interpreted at the 5\% level of significance.

\section{Results}

The estimated age of the cats, reported as median (IQR), was 1.0 (1-2) years for all treatment groups. The weight of the cats was 3.0 (2.2-3.7), 3.1 (2.3-3.6), and 2.8 (2.3-3.5) $\mathrm{kg}$ for MedK, MedKM, and MedKT treatment groups, respectively. Haematocrit and haemoglobin concentration were within clinically normal ranges in all cats. There were no significant differences in signalment or blood parameters among treatment groups.

Table 1. Descriptive statistics and comparisons among treatments for various time frames in minutes from time of injection (T0), except for recovery time, which was considered from the end of surgery to standing. Data reported as median (IQR).

\begin{tabular}{|c|c|c|c|c|}
\hline Time (min) & MedK & MedKM & MedKT & p Value \\
\hline $\begin{array}{l}\text { Induction time (Pedal } \\
\text { reflex loss) }\end{array}$ & $5.6(2.7-8.0)$ & $8.0(5.8-14.9)$ & $7.4(5.1-9.6)$ & 0.125 \\
\hline $\begin{array}{l}\text { Pedal reflex loss to } \\
\text { start of surgery }\end{array}$ & $23.5(20.0-26.1)$ & $20.5(18.8-28.0)$ & $22.7(16.6-30.0)$ & 0.798 \\
\hline Extubation & $63.0(61.8-64.2)$ & $62.8(58.2-64.5)$ & $62.2(61.0-64.0)$ & 0.688 \\
\hline Pedal reflex gained & $69.9(63.0-73.7)$ & $95.2(67.8-107.8)$ & $81.5(71.9-121.5)$ & 0.088 \\
\hline Standing & $160.9(131.7-169.6)$ & $177.9(161.8-214.7)$ & $201.3(155.3-235.1)$ & 0.074 \\
\hline Recovery time & $128.5(95.1-142.8)$ & $142.9(123.4-180.2)$ & $166.4(123.1-210.0)$ & 0.137 \\
\hline
\end{tabular}

$\mathrm{IQR}=$ interquartile range. 
Induction phase

Induction was characterised as being rapid and excitement-free and event times did not vary among treatment groups (Table 1). Median (IQR) time for induction for all three treatment groups was 6.88 (4.90-8.65) minutes. Increased agitation or activity before recumbency, and presence of nausea and emesis were not different among groups (Table 2).

Table 2. Clinical findings during the induction, maintenance, and recovery phase of general anaesthesia. Data reported as number of cats within treatment group, where 10 cats were assigned to each treatment group.

\begin{tabular}{|c|c|c|c|c|}
\hline Clinical parameter & MedK & MedKM & MedKT & p Value \\
\hline \multicolumn{5}{|l|}{ Induction phase } \\
\hline Agitation & 3 & 6 & 5 & 0.392 \\
\hline Nausea & 3 & 5 & 4 & 0.659 \\
\hline Emesis & 1 & 1 & 1 & 1.0 \\
\hline \multicolumn{5}{|l|}{ Maintenance phase } \\
\hline Alfaxalone rescue & 0 & 2 & 0 & 0.117 \\
\hline Partial laryngospasm & 3 & 4 & 3 & 0.861 \\
\hline $\mathrm{O}_{2}$ rescue & 6 & 6 & 7 & 0.866 \\
\hline Apneustic breathing pattern & 2 & 2 & 4 & 0.506 \\
\hline $2^{\circ} \mathrm{AV}$ blocks & 6 & 4 & 2 & 0.189 \\
\hline \multicolumn{5}{|l|}{ Surgery Phase } \\
\hline Isoflurane rescue & 3 & 3 & 4 & 0.861 \\
\hline \multicolumn{5}{|l|}{ Recovery phase } \\
\hline Emesis & 3 & 0 & 0 & 0.036 \\
\hline
\end{tabular}


Maintenance phase

Alfaxalone administration during tracheal intubation, glottis activity during intubation, $\mathrm{SpO}_{2}$ readings, isoflurane administration during surgery, the occurrence of apneustic breathing pattern or of second-degree atrioventricular blocks were not significantly different among groups (Table 2).

Table 3. Descriptive statistics and comparison among treatments for blood gas values, $\mathrm{PE}^{\prime} \mathrm{CO}_{2}, \mathrm{SpO}_{2}$, $\mathrm{OE}$ ratio, and $\mathrm{P}(\mathrm{A}-\mathrm{a}) \mathrm{O}_{2 .}$. Data reported as median (IQR).

\begin{tabular}{|c|c|c|c|c|c|c|c|c|}
\hline \multicolumn{2}{|c|}{ Variable } & \multicolumn{2}{|r|}{ MedK } & \multicolumn{2}{|c|}{ MedKM } & \multicolumn{2}{|c|}{ MedKT } & \multirow{2}{*}{$\begin{array}{c}\text { p Value } \\
0.560\end{array}$} \\
\hline $\mathrm{PaO}_{2}$ & $(\mathrm{mmHg})$ & 68 & $(58-74)$ & 65 & $(60-72)$ & 66 & $(55-68)$ & \\
\hline & $(\mathrm{kPa})$ & 9.1 & $(7.8-9.9)$ & 8.7 & $(8.0-9.6)$ & 8.7 & $(7.3-9.1)$ & \\
\hline \multirow[t]{2}{*}{$\mathrm{PaCO}_{2}$} & $(\mathrm{mmHg})$ & 36 & $(33-37)$ & 35 & $(33-37)$ & 36 & $(35-37)$ & 0.628 \\
\hline & $(\mathrm{kPa})$ & 4.8 & $(4.4-5.0)$ & 4.6 & $(4.4-4.9)$ & 4.8 & $(4.7-5.0)$ & \\
\hline $\mathrm{pH}$ & & 7.326 & $(7.32-7.34)$ & 7.325 & $(7.32-7.34)$ & 7.344 & $(7.31-7.37)$ & \\
\hline \multirow[t]{2}{*}{$\mathrm{PE}^{\prime} \mathrm{CO}_{2}$} & $(\mathrm{mmHg})$ & 41 & $(39-44)$ & 43 & $(42-47)$ & 41 & $(39-45)$ & 0.334 \\
\hline & $(\mathrm{kPa})$ & 5.4 & $(5.1-5.8)$ & 5.7 & $(5.5-6.3)$ & 5.4 & $(5.2-6.0)$ & \\
\hline $\mathrm{SpO}_{2}$ & $(\%)$ & 88 & $(86-93)$ & 89 & $(88-92)$ & 88 & $(85-91)$ & 0.627 \\
\hline $\mathrm{P}(\mathrm{A}-\mathrm{a}) \mathrm{O}_{2}$ & $(\mathrm{mmHg})$ & 14 & $(14-22)$ & 21 & $(15-22)$ & 18 & $(16-27)$ & 0.717 \\
\hline
\end{tabular}


Table 4. Descriptive statistics and comparison among treatments for variables associated with autonomic responses to surgical stimulation: heart rate $(\mathrm{HR})$, respiration rate $\left(f_{R}\right)$ and systolic arterial blood pressure (SABP) for baseline and five critical time points during surgery reported as median (IQR).

\begin{tabular}{|c|c|c|c|c|c|c|c|}
\hline Variable & Group & Baseline & HP & SC1 & TP1 & SC2 & TP2 \\
\hline \multirow{6}{*}{$\begin{array}{l}\text { HR } \\
\text { (beats } \\
\text { minute }^{-1} \text { ) }\end{array}$} & MedK & 126 & 127 & 125 & 123 & 128 & 136 \\
\hline & & $(116-132)$ & (116-134) & $(113-130)$ & $(112-131)$ & $(115-136)$ & $(117-142)$ \\
\hline & MedKM & 119 & 124 & 115 & 127 & 125 & 132 \\
\hline & & (99-133) & $(103-131)$ & $(95-133)$ & $(102-135)$ & (110-133) & $(128-137)$ \\
\hline & MedKT & 128 & 132 & 129 & 128 & 131 & 130 \\
\hline & & $(125-140)$ & $(118-143)$ & $(121-140)$ & $(124-142)$ & $(123-141)$ & $(127-140)$ \\
\hline \multirow{6}{*}{$\begin{array}{l}f_{R} \\
\text { (breaths } \\
\text { minute }^{-1} \text { ) }\end{array}$} & MedK & 18 & 16 & 20 & 23 & 25 & 22 \\
\hline & & $(10-25)$ & $(9-23)$ & $(11-25)$ & $(14-28)$ & $(16-29)$ & $(16-29)$ \\
\hline & MedKM & 19 & 21 & 19 & 22 & 19 & 21 \\
\hline & & $(15-24)$ & $(11-22)$ & $(15-26)$ & $(16-25)$ & $(15-26)$ & $(15-28)$ \\
\hline & MedKT & 16 & 15 & 17 & 19 & 17 & 20 \\
\hline & & $(11-22)$ & $(10-18)$ & $(12-24)$ & $(10-25)$ & $(10-21)$ & $(11-22)$ \\
\hline \multirow{6}{*}{$\begin{array}{l}\text { SABP } \\
(\mathrm{mmHg})\end{array}$} & MedK & 151 & 157 & 152 & 152 & 151 & 157 \\
\hline & & $(122-161)$ & (136-167) & $(128-161)$ & $(126-164)$ & (124-168) & $(125-174)$ \\
\hline & MedKM & 149 & 155 & 148 & 151 & 154 & 154 \\
\hline & & $(135-153)$ & $(139-160)$ & $(136-154)$ & $(140-159)$ & (144-160) & $(144-174)$ \\
\hline & MedKT & 162 & 165 & 167 & 165 & 163 & 163 \\
\hline & & $(142-171)$ & $(143-177)$ & $(133-175)$ & $(138-178)$ & $(138-170)$ & (139-174) \\
\hline
\end{tabular}



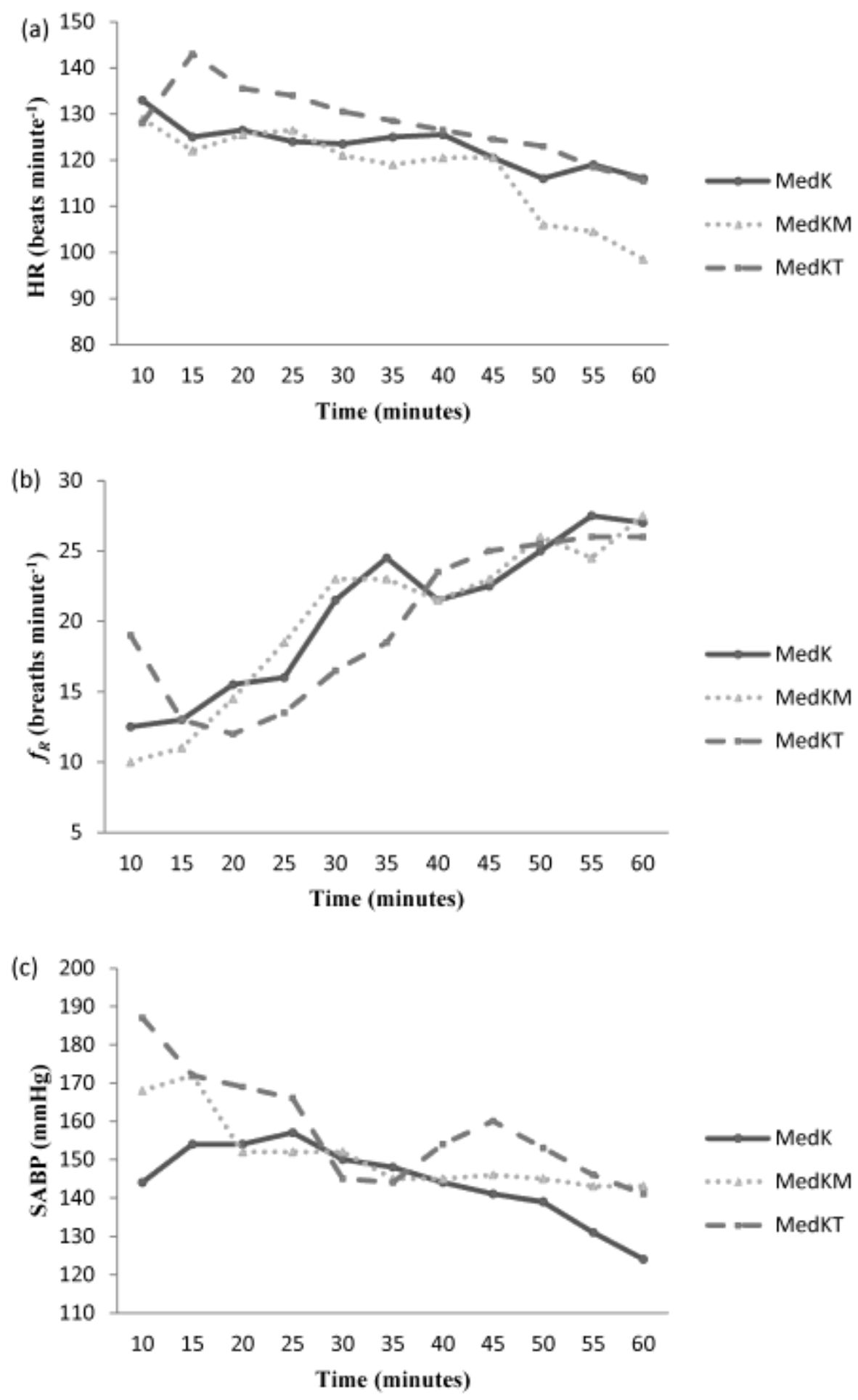

Figure 1 Descriptive statistics and comparison among treatments for median values over time (minutes) for heart rate (HR) a), respiratory rate (b), and systolic arterial blood pressure (c) from 10 to $60 \mathrm{~min}$ after T0. 
All cats requiring oxygen rescue were due to a low $\mathrm{SpO}_{2}$ reading except for one in MedKT group that had a low $\mathrm{PaO}_{2}$ value despite having an above normal $\mathrm{SpO}_{2}$ reading. There were no significant differences among treatment groups for arterial blood gases, $\mathrm{pH}, \mathrm{PE}^{\prime} \mathrm{CO}_{2}$, $\mathrm{SpO}_{2}$, and $\mathrm{P}(\mathrm{A}-\mathrm{a}) \mathrm{O}_{2}$ (Table 3$)$. The $\mathrm{SpO}_{2}$ reading increased by (mean $\left.\pm \mathrm{SD}\right) 3 \pm 1 \%$ when the side-stream gas analyser sampling line was disconnected from the ET tube and returned to the previous value after reconnection.

During maintenance of anaesthesia (excluding times of surgical stimulation), cats in all treatment groups displayed similar cardiovascular $(\mathrm{HR}, \mathrm{SABP})$ and respiratory $\left(f_{\mathrm{R}}\right)$ parameters returning to normal physiological values after 25 minutes from T0 (Fig 1). Surgery had a significant $(\mathrm{p}<0.01)$ effect on $\mathrm{HR}, \mathrm{SABP}$ and $f_{\mathrm{R}}$ causing an increase in these parameters in all treatment groups similarly (Table 4).

\section{Recovery phase}

Recovery was characterised as being long and excitement-free. None of the cats required emergency reversal of any drugs. Three cats in MedK group had emesis during recovery compared to no cats in the other treatment groups $(\mathrm{p}=0.03)$. Duration of apparent euphoria was longer in the MedKM ( $\mathrm{p}=0.003)$ and MedKT $(\mathrm{p}=0.006)$ groups compared to MedK group. The proportion of cats with mydriasis was different among groups $(\mathrm{p}=0.046)$. MedKM and MedKT treated cats had mydriatic pupils up to 8 hours post-injection compared to 4 hours in MedK cats.

\section{Pain scoring}

Multidimensional composite pain scores and VAS scores did not vary among groups (Table 5). The VAS scores recorded were 0 in almost all cats, except in two cats assigned to MedK and 
Table 5. Descriptive statistics and comparisons among treatments for multidimensional composite pain scores evaluated at different time points during recovery, expressed as time after injection of the drugs (T0). Data reported as median (IQR).

\begin{tabular}{|c|c|c|c|c|}
\hline Time (Hour) & MedK & MedKM & MedKT & p Value \\
\hline 3 & $4(3-5)$ & $5(4-5)$ & $5(4-5)$ & 0.644 \\
\hline 4 & $2(1-4)$ & $3(2-4)$ & $4(2-5)$ & 0.375 \\
\hline 5 & $2(1-2)$ & $2(1-3)$ & $2(1-3)$ & 0.982 \\
\hline 6 & $1(0-1)$ & $1(0-1)$ & $1(0-2)$ & 0.809 \\
\hline 7 & $0(0-1)$ & $0(0-1)$ & $1(0-1)$ & 0.730 \\
\hline 8 & $0(0-1)$ & $0(0-0)$ & $0(0-0)$ & 0.782 \\
\hline
\end{tabular}

$\mathrm{IQR}=$ interquartile range.

MedKM groups and one assigned to the MedKT group that obtained a score of less than 30. Cats had a significantly higher multidimensional composite pain score when they were sedated compared to when they were non-sedated $(\mathrm{p}<0.001)$. Sedation was noticed in 9 cats from each treatment group 4 hours after T0; in 5 cats from the MedKM, 5 cats from the MedKT and 1 cat from the MedK groups 6 hours after T0; and in 2 cats from the MedKT group 8 hours after T0 (p = 0.190). Mechanical threshold measurements also did not vary among groups; however, they varied over time differently by treatment $(\mathrm{p}<0.001)$. Mechanical threshold values within the MedK group returned to baseline more rapidly than in MedKM and MedKT groups (Fig 2). 


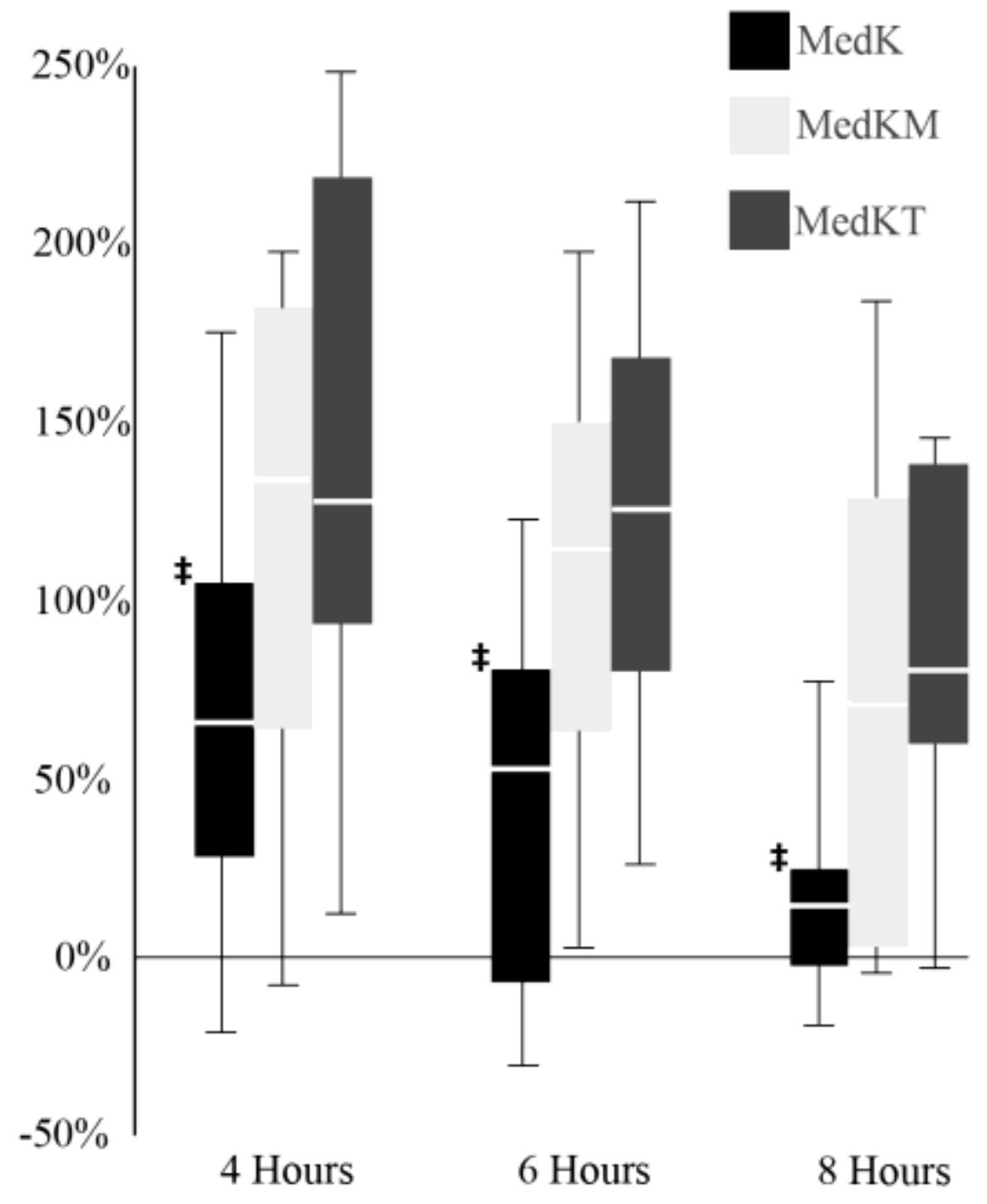

Figure 2 Descriptive statistics and comparison among treatments for percent (\%) change from baseline (0\%) von Frey values over time (hours) from TO. Box plot and whiskers represent median (IQR) and range, respectively. ${ }^{\ddagger}$ Statistically significant finding where MedK return to baseline values faster compared to MedKM and MedKT.

\section{Discussion}

The present study demonstrated that the IM administration of medetomidine and ketamine together, or in further combination with either morphine or tramadol induced a similar, rapid and stable state of general anaesthesia associated with infrequent arousal requiring isoflurane rescue during surgery. All combinations provided adequate intra-operative analgesia for orchiectomy in 
domestic cats. Clinically significant findings included a moderate hypoxemia detected on arterial blood gas analysis and $\mathrm{SpO}_{2}$ in all treatment groups; and an increased mechanical stimulus threshold for a longer duration within the MedKM and MedKT treatment groups despite having a descriptively higher multidimensional composite pain score when compared to the MedK treatment group. Other clinically relevant findings included the need for alfaxalone rescue to allow endotracheal intubation in 2 cats from the MedKM treatment group and emesis during the recovery phase of 3 cats from the MedK treatment group.

Ketamine has been used together with medetomidine for induction and maintenance of general anaesthesia in cats for many years, a variety of doses of both ketamine and of medetomidine having been advocated, with or without the further addition of an opioids, and/or a benzodiazepine (Verstegen et al 1989, Clarke et al, 2014). Morphine administered at $0.2 \mathrm{mg} \mathrm{kg}^{-1}$ in combination with ketamine and medetomidine has been studied in cats using noxious stimulation by applying a Pean intestinal clamp to the metatarsus and tail base (Wiese et al. 2007). Tramadol administered alone at $2 \mathrm{mg} \mathrm{kg}^{-1}$ either subcutaneously (Brondani et al. 2009) or IV (Cagnardi et al. 2011) did not cause unacceptable cardiovascular side effects and provided adequate analgesia for gonadectomy in domestic cats. The present study used tramadol administered IM at the same dose, but additionally combined with ketamine and medetomidine.

Low lingual $\mathrm{SpO}_{2}$ readings have been recorded in studies using combinations of ketamine $\left(10 \mathrm{mg} \mathrm{kg}^{-1}\right)$, medetomidine $\left(100 \mu \mathrm{g} \mathrm{kg}^{-1}\right)$ and buprenorphine $\left(10 \mu \mathrm{g} \mathrm{kg}^{-1}\right)$ (Harrison et al. 2011) or tiletamine/zolazepam $\left(4 \mathrm{mg} \mathrm{kg}^{-1}\right)$, ketamine $\left(6.5 \mathrm{mg} \mathrm{kg}^{-1}\right)$ and xylazine $\left(1.65 \mathrm{mg} \mathrm{kg}^{-}\right.$ ${ }^{1}$ ) (Cistola et al. 2004) in cats. These studies postulated hypoxemia as the cause of the low $\mathrm{SpO}_{2}$ 
readings, but did not perform arterial blood gas analysis to confirm the low $\mathrm{PaO}_{2}$, so it was possible that intense peripheral vasoconstriction due to medetomidine and xylazine could have influenced the accuracy of the lingual $\mathrm{SpO}_{2}$ readings (Harrison et al. 2011; Cistola et al. 2004). A reading of $88 \%$ on the pulse oximeter used in the present study was associated with a $\mathrm{PaO}_{2}$ of 67 $(8.9 \mathrm{kPa}) \mathrm{mmHg}$, which is consistent with studies of feline oxygen haemoglobin saturation (Cambier et al. 2004). This finding suggests that the pulse oximeter was able to accurately detect low oxygen haemoglobin saturation despite the intense peripheral vasoconstriction. The present study observed a clinically significant increase in the $\mathrm{SpO}_{2}$ reading once the side-stream sampling line was removed from the endotracheal tube. This could suggest that a sampling rate of $200 \mathrm{~mL}$ minute ${ }^{-1}$ was high enough to alter the inspired alveolar gas mixture. The mean calculated minute volume for the 30 cats was $609 \mathrm{~mL}$ minute ${ }^{-1}$, which means the sampling line was extracting $33 \%$ of the minute volume of gases for analysis. Retrospectively, the sampling rate should have been adjusted to $50 \mathrm{~mL}$ minute ${ }^{-1}$ to lessen the effects on the pulse oximeter reading and perhaps the arterial blood gas results.

The $\mathrm{PaO}_{2}$ of $67 \mathrm{mmHg}(8.9 \mathrm{kPa})$ was lower than expected based on predicted $\mathrm{PaO}_{2}$ levels. The predicted $\mathrm{P}_{\mathrm{A}} \mathrm{O}_{2}$ based on the altitude and ambient temperature in theatre was (mean \pm SD) $85 \pm 4 \mathrm{mmHg}(11.3 \pm 0.5 \mathrm{kPa})$. The expected $\mathrm{PaO}_{2}$ levels may be slightly lower than this value, often in the order of 3.75 to $15 \mathrm{mmHg}$ less due to physiological right-to-left shunting (Baylis et al. 2009). The possible causes for the lower than anticipated $\mathrm{PaO}_{2}$ values detected within the present study include: 1) the side-stream sampling rate being excessively high thus decreasing the minute volume of gases within the lung; 2$)$ the assumed $\mathrm{P}(\mathrm{A}-\mathrm{a}) \mathrm{O}_{2}$ gradient was slightly higher compared to the normal expected range of 3.75 to $15 \mathrm{mmHg}$ (Baylis et al. 2009), 
which suggests increasing respiratory dead space, and/or a ventilation-perfusion (V/Q) mismatch.

The $\mathrm{PaCO}_{2}$ and $\mathrm{PE}^{\prime} \mathrm{CO}_{2}$ values were within expected physiological range and did not suggest ventilatory depression, as has been observed in previous studies (Wiese et al. 2007; Harrison et al. 2011; Cistola et al. 2004) using similar drug combinations. Surgical stimulation significantly increased the $\mathrm{HR}, \mathrm{SABP}$ and $f_{\mathrm{R}}$ which suggests that the plane of anaesthesia was allowing autonomic responses to occur in response to surgery. Isoflurane rescue was needed in some cats, which is similar to findings of previous studies using similar combinations (Wiese et al. 2007; Harrison et al. 2011; Cistola et al. 2004). The long time frame from induction to start of surgery (mean \pm SD: $22.2 \pm 1.6$ minutes) was suspected to have contributed to arousal during surgery due to waning of the clinical effects of the drugs (Cagnardi et al. 2011; Hanna et al. 1988; Pypendop et al. 2008; Salonen 1989; Taylor et al. 2001). This was also the time when the monitored parameters ( $\mathrm{HR}, \mathrm{SABP}$ and $f_{\mathrm{R}}$ ) returned to normal expected values, which supports this hypothesis. Alfaxalone rescue was required in 2 cats within the MedKM group, which has been described in a previous study using the same combination at a different ketamine dose ( 5 $\mathrm{mg} \mathrm{kg}^{-1}$ ) (Wiese et al. 2007). These findings suggest that not all cats may not reach a deep enough surgical plane of anaesthesia with the doses administered in the present study.

In this study, recoveries were long. Although they could have been speeded up by antagonism of the medetomidine, the disadvantage of such antagonism is that analgesia is also antagonised, and ketamine induced convulsive activity sometimes occurs. In contrast, in the current study the recovery phase was excitement-free with all combinations. 
Various methods have been used to detect mechanical (Brondani et al. 2009; Castro et al. 2009; Steagall et al. 2008) analgesic properties of drugs in cats. In the present study, a von Frey mechanical threshold device was applied to the mid-thorax as opposed to a tension bracelet (Steagall et al. 2008; Steagall et al. 2007) applied to the distal limb used in previous studies. A rigid tip von Frey device, as used in the present study, has been successfully used to evaluate the antinociceptive effects of morphine in a pharmacodynamic modelling study in dogs (KuKanich et al. 2005). The baseline readings were aimed at gauging the cats' natural responses to this type of stimulation and to determine the individual end-points for the cats. The pre-determined endpoint was then used for that individual cat throughout all measurements post-injection. The von Frey device was found to be useful to assess length of reduced nociception to mechanical stimulation in this study and proved to be more sensitive than the multidimensional composite and the VAS pain scales to detect differences between treatments. Cats in the MedKM and MedKT groups had higher mechanical thresholds for longer compared with the MedK group, which was probably due to the analgesic effects of the opioids used within the combinations. No statistically significant data was found comparing the level of sedation and the increased mechanical thresholds among the treatment groups. However, a previous study in dogs using the von Frey device was able to detect a difference in mechanical nociception threshold between sedated and un-sedated dogs (KuKanich et al. 2005). In the current study, the mid-lateral thorax proved to be an anatomical area that most cats tolerated well allowing a steady application of pressure required by the device to detect mechanical stimulation thresholds.

The multidimensional composite scores were higher during the first few hours and gradually decreased over time, which is contrary to the anticipated results. It was anticipated that 
as the analgesic effects of the drugs wore off then the score would increase due to the pain. It would appear the scoring system tended to penalise sedated cats due to their quieter nature (Brondani et al. 2011), making it difficult to evaluate true pain in sedated cats using this scale. None of the pain scoring systems used in the study could detect any difference between treatment groups, which suggests that the systems are not sensitive enough to detect pain or that the pain was not severe enough to allow detection.

Limitations of the study include a small sample size for some analyzed parameters (e.g. pain scoring systems, sedation scores) unrelated to the sample size calculation, the high sidestream sampling rate of the gas analyser, orchiectomy not being painful enough to detect the analgesic effects of the combinations or to detect significant pain using the pain scoring systems.

In conclusion, the three drug combinations used in the present study provided similar anaesthetic, analgesic and cardiorespiratory effects in cats undergoing orchiectomy. Rescue induction and maintenance agents should be readily available when these drug combinations are used for surgical anaesthesia in cats. Hypoxaemia is a concern with all the studied combinations and oxygen supplementation is recommended.

\section{Acknowledgements}

The authors would like to thank the University students and staff members well as Wollies animal project (local outreach welfare organisation) for participating in the study. The primary author would like to thank all co-authors for their guidance and financial support in the study as well as Dr Lynette Bester for assisting in data collection when needed. 


\section{References}

Baylis C, Till C (2009) Interpretation of arterial blood gases. Surgery (Oxford) 27, 470-474.

Brodbelt DC, Pfeiffer DU, Young LE et al. (2007) Risk factors for anaesthetic-related death in cats: results from the confidential enquiry into perioperative small animal fatalities (CEPSAF). Br J Anaesth 99(5), 617-623.

Brodbelt DC, Blissitt KJ, Hammond RA et al. (2008) The risk of death: the confidential enquiry into perioperative small animal fatalities. Vet Anaesth Analg, 35(5) 365-373.

Brondani JT, Loureiro Luna SP, Beier SL et al. (2009) Analgesic efficacy of perioperative use of vedaprofen, tramadol or their combination in cats undergoing ovariohysterectomy. J Feline Med Surg $11,420-429$.

Brondani JT, Luna SP, Padovani CR (2011) Refinement and initial validation of a multidimensional composite scale for use in assessing acute postoperative pain in cats. Am J Vet Res 72, 174-183.

Cagnardi P, Villa R, Zonca A et al. (2011) Pharmacokinetics, intraoperative effect and postoperative analgesia of tramadol in cats. Res Vet Sci 90, 503-509.

Cambier C, Wierinckx M, Clerbaux T et al. (2004) Haemoglobin oxygen affinity and regulating factors of the blood oxygen transport in canine and feline blood. Res Vet Sci 77, 83-88.

Castro DS, Silva MFA, Shih AC et al. (2009) Comparison between the analgesic effects of morphine and tramadol delivered epidurally in cats receiving a standardized noxious stimulation. Journal of Feline Medicine \& Surgery 11, 948-953.

Cinel D, Markwell K, Lee R et al. (1991) Variability of the respiratory gas exchange ratio during arterial puncture. Am Rev Respir Dis 143, 217-218.

Cistola AM, Golder FJ, Centonze LA et al. (2004) Anesthetic and physiologic effects of tiletamine, zolazepam, ketamine, and xylazine combination (TKX) in feral cats undergoing surgical sterilization. Journal of Feline Medicine \& Surgery 6, 297-303.

Clarke KW, Trim CM , Hall LW (2014) Veterinary Anaesthesia. $11^{\text {th }}$ Edition. Chapter 16, Anaesthesia of the Cat, pgs 503-520 - Saunders, Elsevier, London.

Hanna RM, Borchard RE, Schmidt SL (1988) Pharmacokinetics of ketamine $\mathrm{HCl}$ and metabolite I in the cat: a comparison of i.v., i.m., and rectal administration. J Vet Pharmacol Ther 11, 84-93.

Harrison KA, Robertson SA, Levy JK et al. (2011) Evaluation of medetomidine, ketamine and buprenorphine for neutering feral cats. Journal of Feline Medicine \& Surgery 13, 896-902.

Henik RA, Dolson MK, Wenholz LJ (2005) How to Obtain a Blood Pressure Measurement. Clin Tech Small Anim Pract 20, 144-150. 
KuKanich B, Lascelles BD, Papich MG (2005) Assessment of a von Frey device for evaluation of the antinociceptive effects of morphine and its application in pharmacodynamic modeling of morphine in dogs. Am J Vet Res, 66(9), 1616-1622.

Pypendop BH, Ilkiw JE (2008) Pharmacokinetics of tramadol, and its metabolite O-desmethyl-tramadol, in cats. J Vet Pharmacol Ther 31, 52-59.

Salonen JS (1989) Pharmacokinetics of medetomidine. Acta Vet Scand Suppl 85, 49-54.

Steagall PV, Taylor PM, Brondani JT et al. (2007) Effects of buprenorphine, carprofen and saline on thermal and mechanical nociceptive thresholds in cats. Vet Anaesth Analg 34, 344-350.

Steagall PVM, Taylor PM, Brondani JT et al. (2008) Antinociceptive effects of tramadol and acepromazine in cats. Journal of Feline Medicine \& Surgery 10, 24-31.

Taylor PM, Robertson SA (2004) Pain management in cats - past, present and future. Part 1 . The cat is unique. Journal of Feline Medicine \& Surgery 6, 313-320.

Taylor PM, Robertson SA, Dixon MJ et al. (2001) Morphine, pethidine and buprenorphine disposition in the cat. J Vet Pharmacol Ther 24, 391-398.

Verstegen J, Fargetton X, Ectors F. (1989) Medetomidine/ketamine anaesthesia in cats. Acta Vet Scand Suppl. 1989;85:117-23.

Wiese AJ, Muir WW (2007) Anaesthetic and cardiopulmonary effects of intramuscular morphine, medetomidine and ketamine administered to telemetered cats. Journal of Feline Medicine \& Surgery 9 , 150-156. 